\title{
Aplicação de um Índice de Qualidade de Água para o sistema de abastecimento público
}

Application of a Water Quality Index to the public water supply system

\author{
R. A. S. Silva' ${ }^{1}$ I. N. F. Maynard²; A. Q. Barbosa ${ }^{1,2}$; E. C. Tolle 3 ; E. B. \\ Cavalcante $^{1,4,5} ;$ M. N. Marques ${ }^{1,2,5^{*}}$ \\ ${ }^{1}$ Departamento das engenharias da Universidade Tiradentes, CEP 49032-490, Aracaju-Sergipe, Brasil \\ ${ }^{2}$ Programa de pós-graduação em saúde e ambiente da Universidade Tiradentes, CEP 49032-490, Aracaju-Sergipe, \\ Brasil \\ ${ }^{3}$ Controle de qualidade da água na região Centro Oeste de Sergipe - Companhia de Saneamento de Sergipe (Deso), \\ CEP - 49020-380, Aracaju-Sergipe, Brasil \\ ${ }^{4}$ Programa de pós-graduação em engenharia de processos da Universidade Tiradentes, CEP 49032-490, Aracaju- \\ Sergipe, Brasil \\ ${ }^{5}$ Laboratório de tratamento de resíduos e efluentes do Instituto de Tecnologia e Pesquisa
}

*mnmarques63@gmail.com

(Recebido em 07 de julho de 2017; aceito em 28 de setembro de 2017)

\begin{abstract}
Este trabalho tem como objetivo avaliar a qualidade das águas do sistema de abastecimento do município de Rosário do Catete, Sergipe, aplicando o Índice de Qualidade de Água do Conselho Canadense do Ministério do Meio Ambiente (IQA-CCME). Foram coletadas amostras nos períodos seco (fev/16) e chuvoso (jul/16) em quatro pontos, sendo um ponto de água subterrânea, fonte caldas (P1), e três pontos de água tratada, sendo estes: poço Cipó (P2), reservatório (P3) e rede de distribuição (P4). Os valores máximos permitidos (VMP) utilizados como referência foram os estabelecidos pela resolução CONAMA 396/08, para água subterrânea e portaria de potabilidade do MS 2914/11 para água tratada. Na aplicação do IQA-CCME quase todas as amostras foram classificadas como ótimas, exceto, a amostra do P1 no mês de julho de 2016 que foi classificada como ruim, com IQA igual a 46. Deste modo, o IQA CCME apresentou-se como uma ótima ferramenta para avaliar amostras de água de diversas fontes, e neste trabalho, o seu cálculo indicou que a água distribuída a população do município é de excelente qualidade.
\end{abstract}

Palavras-chave: IQA CCME, água bruta, água tratada

This work has as objective a quality evaluation of the water supply system of the municipality of Rosário do Catete, Sergipe, applying the Water Quality Index of the Canadian Council of the Ministrs of the Environment (WQI-CCME). Samples were collected during periods drought (feb/16) and flood (Jul/ 16) in four points, being an underground water point, source Caldas (P1) and three points of treated water, these being: Cipó well (P2), reservoir (P3) and Distribution network (P4). Maximum Permissible Values (MPV) used as reference were those established by CONAMA Resolution 396/08, for groundwater and drinking water management of MS 2914/11 for treated water. In the application of WQI-CCME almost all the samples were classified as excellent, except a sample of P1 in July 2016 that was classified as regular (46). In this way, the WQI-CCME was presented as a great tool to evaluate water samples from several sources, in this work, its calculation indicated that the water distributed to the population of the municipality is of excellent quality Keywords WQI CCME, raw water, drinking water

\section{INTRODUÇÃO}

Nos últimos anos, a humanidade vem sendo atingida por sérios problemas no âmbito ambiental, social e econômico. Devido a esses problemas um dos recursos que mais sofre alteração em suas características é a água, que tem grande importância devido a sua demanda e sua utilização estarem cada vez mais intensa, e consequentemente acabam por impactar com os padrões de conforto e bem-estar da vida moderna [1]. 
Conforme Braga et al. (2005) [2], deve-se ter água no meio ambiente tanto em qualidade quanto em quantidade apropriada para os seus diversos usos, pois a demanda deste recurso vem tornandoo escasso, devido aos diversos tipos de contaminantes existentes.

A água para consumo da população necessita passar por diversos processos para a sua devida potabilização, os quais são realizados em estações de tratamento de água. Conforme Medeiros Filho [3], estes processos são definidos como um serviço público fornecido por um conjunto de obras hidráulicas e instalações que são responsáveis pelo fornecimento de água para atendimento das necessidades da população de uma comunidade.

Para tanto, a avaliação da qualidade da água é um dos instrumentos para a sustentação de uma política de planejamento e gestão de recursos hídricos, funcionando como um sensor de anormalidades que possibilita identificar o processo de uso dos corpos d'água, mostrando seus efeitos sobre as características qualitativas dos recursos hídricos, tendo em vista a subdiação do controle ambiental, com objetivo de garantir a proteção da comunidade sem riscos a sua saúde [4].

Uma ferramenta que pode ser utilizada para caracterizar o atual estado do corpo hídrico é o IQAÍndice de Qualidade da Água [5]. O desenvolvimento do IQA deu-se por apresentar forma simples/resumida os dados de relatórios complexos contendo muita informação a respeito da qualidade de água, tendo seu fundamento através de expressões numéricas que irão definir o seu estado $[6,7]$.

Diversos índices de qualidade de água têm sido desenvolvidos, como o IQA com Peso Aritmético (IQA-PS), IQA da Fundação Nacional da Saúde (IQA-FNS), IQA do Conselho Canadense do Ministério de Meio Ambiente (IQA-CCME), IQA do Oregon (IQA-O) entre outros. Todos estes foram formulados por importantes organizações ambientais internacionais e por isso tem sua utilização defendida por órgãos ambientais [8]. Os mesmos são aplicados para avaliar a qualidade da água em uma área e possibilitar definir seu uso [9]. O desenvolvimento desses índices é baseado, frequentemente, em números de variáveis de qualidade de água que são comparados com seus respectivos valores padrões através das legislações ambientais vigentes [10].

O conselho Canadense do Ministério do Meio Ambiente formulou um índice de qualidade de água (IQA) que é bem aceito devido a sua aplicabilidade. O IQA CCME é uma expressão numérica que consiste essencialmente em três fatores (espectro, frequência e amplitude), sendo um índice considerado "moldável", pois permite a seleção das variáveis a serem utilizadas, para caracterizar o corpo hídrico de forma que se adeque ao seu uso [7,11,12].

A metodologia matemática do IQA CCME é de forma estatística, e é fundamentada na frequência que ocorre as falhas relacionadas às condições da qualidade da água, que são determinadas pelas legislações vigentes para cada tipo de corpo hídrico [13]. Os fatores utilizados no IQA CCME são combinados e relacionados obtendo-se um valor que varia entre 0 a 100 , e descreve a situação da água [14].

Os padrões de potabilidade são necessários para garantir a boa qualidade da água a ser fornecida a população. No Brasil estes padrões são regidos pelo Ministério da Saúde por meio da portaria $\mathrm{n}^{\circ}$ 2914 de 12/12/2011[15], que "estabelece os procedimentos e responsabilidades relacionados ao controle e vigilância da qualidade de água para consumo humano e seu padrão de potabilidade, entre outras providencias". As redes de abastecimento devem fornecer água de acordo com os parâmetros de qualidade exigidos pelas normas da portaria supracitada.

Para água de origem subterrânea tem-se a resolução no 396 de 2008 do Conselho Nacional de Meio Ambiente [16] que "dispõe sobre a classificação das águas subterrâneas e dá outras providencias". Já os corpos de águas superficiais, seus padrões são regidos pela resolução CONAMA 357 de 2005 [17], que "dispõe sobre a classificação dos corpos de água e das suas diretrizes ambientais para o seu enquadramento".

Os parâmetros de qualidade que são avaliados nas legislações citadas anteriormente, em sua maioria, são em grande quantidade e consequentemente suas características são diferentes, dificultando assim a divulgação de informações consolidadas sobre a qualidade da água em um determinado ambiente hídrico. Sendo assim, o IQA CCME apresenta resultados de fácil compreensão para a população leiga em assuntos que detêm de um banco de dados complexo [18].

Sendo assim, o objetivo deste trabalho é aplicar o IQA CCME como instrumento de avaliação da qualidade da água de captação, tratamento e distribuição do município de Rosário do Catete, SE. 


\section{MATERIAL E MÉTODOS}

\section{1- ÁREA DE ESTUDO}

O estudo foi realizado no município de Rosário do Catete, no estado de Sergipe, o qual está contido em duas bacias hidrográficas a bacia do rio Sergipe e bacia do rio Japaratuba. Esta última é a bacia que estão localizados os pontos de captação das amostras de água para abastecimento da população local. A bacia hidrográfica do Rio Japaratuba é considerada a menor bacia hidrográfica do estado com extensão de $1.722 \mathrm{~km}^{2}$, abrangendo uma área de 7,8\% do estado de Sergipe e possui baixo potencial hídrico. Mesmo assim, esta bacia apresenta intensos processos antrópicos como a utilização da água para exploração das atividades minerais (petróleo/ gás e potássio), irrigação de lavouras como a monocultura de cana-de-açúcar, dessedentação de animais, atividades industriais e urbanas $[19,20]$. Portanto, as águas utilizadas para captação, em sua maioria, provem do subsolo e são extraídas através de poços ou por fonte de surgência. Foram definidos 4 pontos de coleta que estão demonstrados na Figura 1 e descritos na Tabela 1.

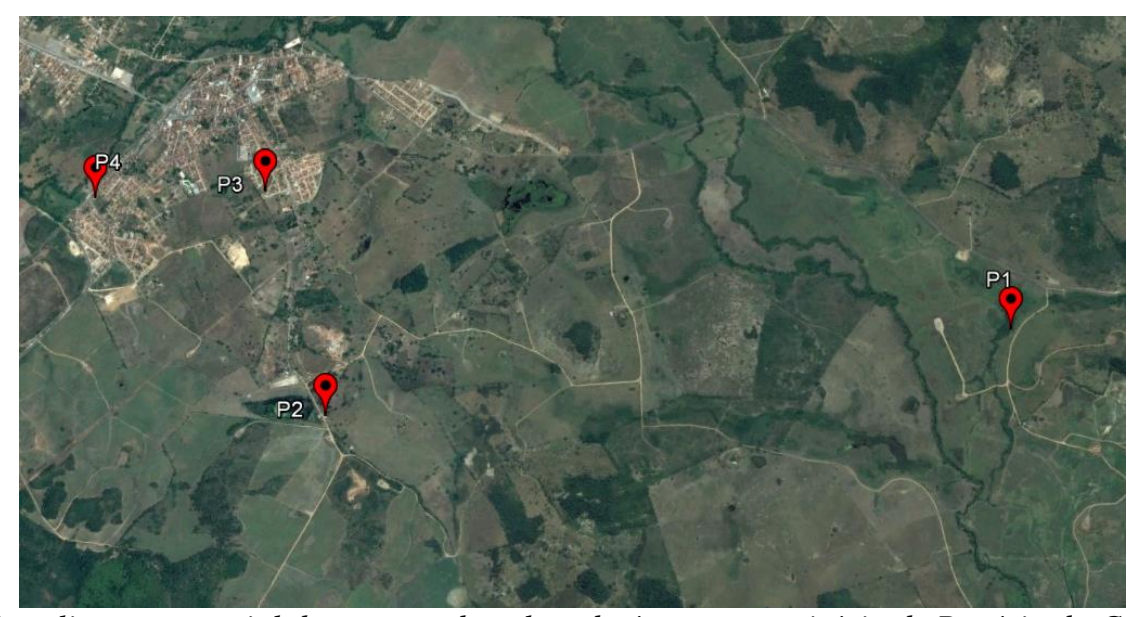

Figura 1: Localização espacial dos pontos de coleta de água no município de Rosário do Catete (Fonte: Google Earth, 2016)

Tabela 1 Identificação e descrição dos pontos de coleta

\begin{tabular}{llll}
\hline Ponto & Descrição & Classificação & Coordenadas Geográficas (WGS 84) \\
\hline P1 & Fonte Caldas & Subterrânea (bruta) & $10^{\circ} 42^{\prime} 20.21^{\prime \prime} \mathrm{S} / 36^{\circ} 59^{\prime} 38.03^{\prime \prime} \mathrm{O}$ \\
\hline P2 & Poço Cipó & Subterrânea (bruta) & $10^{\circ} 42^{\prime} 41.30^{\prime \prime} \mathrm{S} / 37^{\circ} 1^{\prime} 31.84^{\prime \prime} \mathrm{O}$ \\
\hline P3 & Reservatório de água & Tratada & $10^{\circ} 42^{\prime} 4.23^{\prime \prime} \mathrm{S} / 37^{\circ} 1,45.63^{\prime \prime} \mathrm{O}$ \\
\hline P4 & Distribuição & Tratada & $10^{\circ} 42^{\prime} 6.89^{\prime \prime} \mathrm{S} / 37^{\circ} 2^{\prime} 14.67^{\prime \prime} \mathrm{O}$ \\
\hline
\end{tabular}

\section{2- PROCEDIMENTOS}

As coletas foram realizadas em fevereiro/16 e julho/16, totalizando 2 campanhas de coletas, que abrangeram os períodos seco e chuvoso na região, exceto para o poço Cipó ponto P2 que passou a ser analisado depois que a companhia responsável pelo abastecimento o inseriu no sistema de abastecimento da cidade de Rosário do Catete, sendo assim a coleta no período de seca foi realizada em jan/17. Durante as coletas foram utilizados recipientes específicos para cada grupo de parâmetros de qualidade de água. Logo após as coletas as amostras foram condicionadas em caixas térmicas, em seguida levadas para laboratório para analises seguindo as metodologias adotadas no Standard Methods for Examination of Water and Wasterwater [21]. Foram analisados os parâmetros inorgânicos e orgânicos que são contemplados pela Portaria 2914/2011 do Ministério da Saúde, estes são apresentados nas tabelas do Apêndice 1. 


\subsection{1- CÁLCULO DO IQA CCME}

A escolha deste índice deu-se pela sua simplicidade na aplicação e por permitir sua adaptação à verificação da conformidade do corpo hídrico ou amostras de água segundo os parâmetros estabelecidos na legislação vigente. Este índice se baseia em medidas do espectro, da frequência e da amplitude dos valores que estão fora dos padrões estipulados pelas normas: F1 o número de variáveis que apresentaram valores fora dos padrões, (Espectro); F2 a frequência com que valores das análises apresentam-se fora dos padrões, (frequência) e F3 o quanto estes valores se distanciam dos valores padrão, (amplitude). Estes fatores são combinados para produzir um único valor (entre 0 e 100) que descreva a qualidade de água. De acordo com o resultado do IQA a qualidade da água é enquadrada em uma determinada categoria apresentada na Tabela 2. Os cálculos do IQA-CCME podem ser obtidos na literatura referenciada [7].

Para a avaliação do IQA CCME as variáveis (parâmetros de qualidade de água) foram comparadas com os valores máximos permissíveis (VMP) da resolução CONAMA 396/11 [16] para o ponto P1, por ser uma surgência, e com os VMP da portaria de potabilidade 2914/11 [15] para os pontos $\mathrm{P} 2$, P3 e P4. O ponto P2 foi avaliado como água tratada, porque a coleta foi feita após a cloração e na via que ia para o reservatório de distribuição.

Para obtenção do IQA CCME para cada ponto de coleta em todas as campanhas de coleta, foi confeccionada uma planilha de cálculo, utilizando o Microsoft EXCEL. Na Tabela 2 são apresentadas as categorias de acordo os valores obtidos no IQA.

Tabela 2. Escala de valores de IQA e respectiva classificação de acordo com a adotada pelo CCME

\begin{tabular}{ll}
\hline Valor & Categoria \\
\hline $94<\mathrm{IQA} \leq 100$ & Ótima \\
$79<\mathrm{IQA} \leq 94$ & Boa \\
$64<\mathrm{IQA} \leq 79$ & Regular \\
$44<\mathrm{IQA} \leq 64$ & Ruim \\
$\mathrm{IQA} \leq 44$ & Péssima \\
\hline
\end{tabular}

\section{RESULTADOS E DISCUSSÃO}

Foram analisados 82 parâmetros (variáveis) em oito amostras, sendo duas de água bruta (P1) e seis de água tratada (P2, P3 e P4). Destes parâmetros quase todos deram abaixo do limite de quantificação do método como são apresentados nas Tabelas A1-A6 do Apêndice, nas quais constam os resultados de todas as análises.

Obtida as variáveis calculou-se o IQA CCME. Para tanto, elaborou-se planilhas de cálculos utilizando-se o MS-Excel. Os resultados estão apresentados na Tabela 3, contendo o número de testes, quantidade de variáveis, os fatores F1, F2, nse (soma normalizada das excursões), F3 e o IQA para cada ponto de amostragem.

Tabela 3-Valores obtidos para F1, F2, nse, F3 e IQA CCME para as amostras

\begin{tabular}{cclccccccc}
\hline Ponto & Data & Legislação & $\mathbf{N}^{\circ}$ Testes & $\begin{array}{c}\mathbf{N}^{\circ} \\
\text { Variáveis }\end{array}$ & F1 & F2 & nse & F3 & $\begin{array}{c}\text { IQ } \\
\text { A }\end{array}$ \\
\hline P1 & Fev/16 & R.C 396/08 & 79 & 79 & 0 & 0 & 0 & 0 & 100 \\
& Jul/16 & R.C 396/08 & 79 & 79 & 1,8 & 1,8 & 13,4 & 93,1 & 46 \\
\hline P2 & Jul/16 & Port. 2914/11 & 82 & 82 & 4,1 & 4,1 & 29,8 & 96,8 & 99 \\
& Jan/17 & Port. 2914/11 & 82 & 82 & 0 & 0 & 0 & 0 & 100 \\
\hline P3 & Fev/16 & Port. 2914/11 & 82 & 82 & 0 & 0 & 0 & 0 & 100 \\
& Jul/16 & Port. 2914/11 & 82 & 82 & 0 & 0 & 0 & 0 & 100 \\
\hline P4 & Fev/16 & Port. 2914/11 & 82 & 82 & 0 & 0 & 0 & 0 & 100 \\
& Jul/16 & Port. 2914/11 & 82 & 82 & 0 & 0 & 0 & 0 & 100 \\
\hline
\end{tabular}


Nas proximidades dos pontos P1e P2 existem áreas de plantações de cana-de-açúcar, pastagem, criação de gado como também equipamentos utilizados para a extração de petróleo [22,23].

Os resultados do IQA CCME apresentados na Tabela 3 demonstram que ocorreu uma queda na qualidade da água do ponto P1 no período de cheia (jul/16) quando comparado com o período de seca (fev/16). Em fev/16 obteve-se o valor de 100 (Ótima) na escala IQA CCME enquanto que em jul/16 o corpo hídrico apresentou um valor de 46 (Ruim) indicando houve alteração nas características do manancial. A variável que ocasionou esta queda de qualidade na água foi o antimônio $(\mathrm{Sb})$ que ficou acima do VMP $(0,005 \mathrm{mg} / \mathrm{L})$ pela resolução CONAMA 396/11, o valor medido foi 7,28 $\mathrm{mg} \mathrm{Sb} / \mathrm{L}$.

O antimônio é um considerado um semimetal e está presente na crosta terrestre, portanto, a sua presença em alta concentração, neste ponto, pode estar relacionada ao aumento do aporte do material particulado carreado do solo pelas chuvas do referente período [24].

Nas últimas décadas houve um aumento significativo na utilização do antimônio em vários tipos de plásticos (PVC e o PET), e a incineração deste junto com a queima de resíduos fósseis, são lançados ao ar que através do transporte aéreo natural acabam depositando-se em diversos ambientes como o aquático, ocasionando assim a sua contaminação [24, 25,26].

O ponto P2 obteve a classificação excelente para qualidade da água, nos dois períodos analisados, mesmo estando em áreas propicias a contaminação por monocultura de cana-de-açúcar, rebanho bovino ou pela exploração do petróleo. Estes fatores indicam que o aquífero pode estar protegido por camadas de folhelho, como indica o mapa geológico do estado de Sergipe [27].

Os valores do IQA CCME obtidos para os pontos P3 (reservatório) e P4 (rede de distribuição) foram o valor máximo na escala IQA CCME (100), indicando que não houve desvio algum em nenhuma das amostras de água coletadas no período de estudo, mesmos no período de chuva (jun/16) quando a amostra do ponto de captação P1 apresentou alta concentração de antimônio. Este fato indica que o tratamento efetuado pelo sistema de abastecimento local é eficaz, tornando assim essa água apta para consumo humano,pois, a mesma encontra-se dentro dos padrões que são exigidos pela portaria 2914 de 2011 do Ministério da Saúde.

Damo et al (2013) [12] aplicou este IQA para avaliar a qualidade da água de abastecimento da cidade de Pogradec, Albania e obteve resultados semelhantes. Marques et al (2007) [7] compararam este índice com o IQA desenvolvido pela "National Sanitation Foundation" dos Estados Unidos e adotado pela Agencia Nacional de Água (ANA), e concluiram que a classificação obtida pelo IQA CCME é coerente com o IQA, porque os índices não apresentam diferenças entre as classificações.

\section{CONCLUSÃO}

A avaliação da qualidade da água aplicando o IQA CCME no período de estudo permitiu concluir que a água é considerada de excelente qualidade em $87,5 \%$ dos pontos estudados. Embora a amostra da Fonte Caldas no mês de julho de 2016 tenha sido considerada ruim, devido a concentração do antimônio ter excedido o seu valor máximo permitido pela legislação, o processo de tratamento efetuado pela companhia de abastecimento de água local demonstrou-se eficaz, pois os valores do IQA CCME obtidos para água tratada foram excelentes e sem irregularidades em suas características.

O IQA-CCME apresentou-se como uma ótima ferramenta para avaliar a qualidade de água em corpos hídricos com diferentes características e fins (água bruta e tratada), uma vez que este é fundamentado na flexibilidade em incluir ou excluir variáveis de qualidade de água, possibilitando a sua adaptação aos objetivos existentes para cada classe de uso do corpo hídrico.

\section{AGRADECIMENTOS}

Os autores agradecem ao CNPq, a FAPITEC e o Programa de bolsas de iniciação cientifica da UNIT- PROBIC pelas bolsas e apoio financeiro e a DESO pela parceria e colaboração. 


\section{REFERÊNCIAS BIBLIOGRÁFICAS}

1. Peixoto RHPB, Oliveira DA, Araujo CC, Barros EO. Qualidade da água do rio Tocantins a jusante da usina hidreletrica serra da mesa (GO). Congresso Interamericano de Ingenieria sanitaria y ambiental, anais. Cancun, México; 2002.

2. Braga B, Hespanhol I, Conejo JGL, Merzwa JC, Barros MTL, Spencer M, Porto M, Nucci N, Juliano N, Eiger S. Introducer á engenharia ambiental. São Paulo. Prentice Hall; 2005.

3. Medeiros Filho CF. Abastecimento de Água. [Dissertação]. Campina Grande (PB): Universidade Federal da Paraíba em Campina Grande; 2015.

4. Lemos M, Ferreira N, Dias M. Sazonalidade e variabilidade especial da qualidade da água na lagoa do apodi, RN. Rev Bras Eng Agric Ambient. 2010;14(2):155-164, doi:10.1590/s1415-43662010000200006.

5. Khan F, Husain T, Lumb A. Water quality evaluation and trend analysis in selected watersheds of the Atlantic region of Canada. Environ Monit Assess. 2003;88(1-3):221-242, doi:10.1023/A:1025573108513.

6. Bordalo AA, Teixeira R, Wiebe WJ. A water quality index applied to an international shared river basin: The case of the Douro river. Environmental management. Environ Manage. 2006 Dec;38(6):910-920, doi:10.1007/s00267-004-0037-6.

7. Marques MN, Daude LF, Souza RMGL de, Cotrim MEB, Pires MAF. Avaliação de um índice dinâmico de qualidade de água para abastecimento. Um estudo de caso. Rev Exacta. 2007 Jan/Jun;5(1):47-55.

8. Debels P, Figueroa R, Urrutia R, Barra R, Niell X. Evaluation of water quality in the Chillan's river (Central Chile) using physicochemical parameters and modified water quality index. Environ Monit Assess. 2005;110(1-3):301-322, doi:10.1007/s10661-005-8064-1.

9. Lumb A, Halliwell D, Sharma T. Application of CCME Water Quality Index to Monitor Water Quality: A case Study of the Mackenzie River Basin, Canada. Environ Monit Assess. 2006;113(1-3):411-429, doi:10.1007/s10661-005-9092-6.

10. Bordalo AA, Nilsumranchit W, Chalermwat K. Water quality and uses of Bagpakong river (Eastern Thailand). Water Res. 2001 Oct;35(15):3635-42.

11. Hulya B. Utilization of the water quality index method as a classification tool. Environ Monit Assess. 2010 Aug;167(1-4):115-24, doi:10.1007/s10661-009-1035-1.

12. Damo R, Icka P. Evaluation of water quality index for drinking water. University of Korça, Agronomy Department, Albania.

13. CCME, Canadian Council of Ministers of the Environment. Canadian water quality guidelines for the protection of aquatic life: CCME water quality index 1.0, technical report. Canada 2001. Disponível em: http://www.ccme.ca/files/resources/calculators/wqi\%20user's20manual\%20(en).pdf. Acesso: 06 de agosto de 2016.

14. Khan AA, Tobin A, Paterson R, Khan H, Warren R. Application of CCME Produces for deriving sitespecific water quality guideline for the CCME water quality index. Water quality RES J Canada. 2005;40(4):448-456.

15. Brasil, Ministério da saúde, Brasília, Portaria n²914, de 12 de dezembro de 2011. Dispõe sobre os procedimentos de controle e vigilância da qualidade da água para o consume humano e seu padrão de potabilidade. Disponível: http://bvsms.saude.gov.br/bvs/saudelegis/gm/2011/prt2914_12_12_2011.html. Acesso: 20 de março de 2016.

16. Conselho Nacional de Meio Ambiente. Resolução de n⿳306, de 03 de abril de 2008. Dispõe sobre a classificação e diretrizes ambientais para o enquadramento das águas subterrâneas e dá outras providencias. Disponível em: http://www.mma.gov.br/port/conama/legiabre.cfm?codlegi=459. Acesso: 20 de março de 2016.

17. Conselho Nacional de Meio Ambiente. Resolução de $\mathrm{n}^{\circ} 357$ de 17 d março de 2005. Dispõe sobre a classificação dos corpos d1agua e diretrizes ambientais para o seu enquadramento, bem como estabelece as condições e padrões de lançamento de efluentes. Disponível: http//www.mma.gov.br/port/conama/legiabre.cfm?codlegi=459. Acesso: 20 de março de 2016.

18. Porto MFA, Sergio JL, Nogueira VP. Hidrologia Ambiental. São Paulo: Edusp; 1991. Cap. 1, p. 27-65.

19. Jica/SEPLANTEC. Estudo sobre o desenvolvimento dos recursos hidricos no Estado de Sergipe na República Federativa do Brasil. Japan International Cooperation Agency. Secretaria de Estado do Planejamento e da Ciência e Tecnologia. Aracaju; 2000.

20. Pantaleão SM. Impacto genotóxico de poluentes químicos presentes na água e sedimentos do rio Japaratuba. Tese (doutorado) - Programa de Pós-Graduação em Genética e Bioquímica, Universidade Federal de Uberlândia. Uberlândia, 2006.

21. APHA, AWWA, WEF. Standard Methods for examination of water and wastewater. $22^{\text {nd }}$ ed. Washington: American Public Health Association; 2012, 1360 p. ISBN 978-087553-013-0. 
22. SEMARH. Secretaria de Estado do Meio Ambiente e dos Recursos Hídricos. Execução dos serviços para a elaboração do plano da bacia hidrográfica do rio Japaratuba, diagnostico integrado, 2010.

23. EMDAGRO. Empresa de Desenvolvimento Agropecuário de Sergipe. Informações básicas municipais do município de Rosário do Catete. Governo de Sergipe. 2008.

24. Fillela M, Belzile N, Chen YW. Antimony in the environmental: a review focused on natural waters-I. Ocurrence, Earth- Science Review. 2002;57:125-176, doi:10.1016/s0012-8285(01)00070-8.

25. Shotyk W, Krachler M. Chen B. Contamination of Canadian and European bottled waters with antimony from PET containers. Environmental. Science Technol. 2006;8:288-292, doi:10.1039/b517844b.

26. Krachler M, Zheng J, Koerner R, Zdanowicz C. Fisher D, Shotyk W. Increasing atmospheric antimony contamination in the northern hemisphere: snow and ice evidence from Devon Island, Arctic Canada. J. Environ Monit. 2005 Dec;7(12):1169-76, doi:10.1039/b509373b.

27. CPRM- Serviço Geológico do Brasil, Mapa Geológico do Estado de Sergipe. Programa levantamentos geologico básicos do Brasil. Brasília; 1998. 